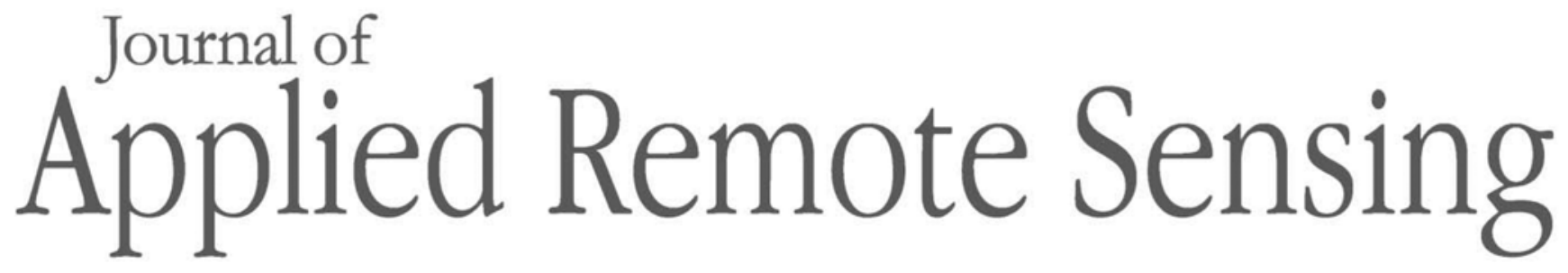

RemoteSensing.SPIEDigitalLibrary.org

\title{
Special Section Guest Editorial: Optics in Atmospheric Propagation and Adaptive Systems
}

Karin Stein

Szymon Gładysz

Christian Eisele

Vladimir Lukin in Atmospheric Propagation and Adaptive Systems," J. Appl. Remote Sens. 12(4), 042401 (2018), doi: $10.1117 / 1 . J R S .12 .042401$. 


\title{
Special Section Guest Editorial: Optics in Atmospheric Propagation and Adaptive Systems
}

\author{
Karin Stein, ${ }^{a}$ Szymon Gładysz, ${ }^{a}$ Christian Eisele, ${ }^{a}$ and Vladimir Lukin ${ }^{b}$ \\ ${ }^{a}$ Fraunhofer Institute for Optronics, System Technologies and Image Exploitation, \\ Department Signatorics, Ettlingen, Germany \\ ${ }^{b}$ V.E. Zuev Institute of Atmospheric Optics, Siberian Branch of the Russian Academy of \\ Science, Tomsk, Russia
}

The use of sensors for active and passive remote sensing of the Earth and its atmosphere, for freespace laser communications, and for high-resolution imaging of ground-based and airborne objects encompasses fields of growing interest for both civilian and military applications. Many applications ask for space-to-ground (e.g. remote sensing satellites) or ground-tospace (e.g. astronomical telescopes) imaging systems with ever-improving spectral and/or spatial resolution, working in spectral regions from UV to radio.

All of these systems have in common that their performance is often not limited by system design but by effects caused by the long propagation of the signal through the atmosphere, which acts as an absorbing, scattering, and radiating random medium. A profound understanding of these effects is needed for an accurate analysis of system performance and the correct interpretation of measurement results.

Performance of operational systems may be improved by implementation of modern methods allowing for the reduction of effects associated with signal propagation. These include postprocessing algorithms and real-time hardware, making the system adaptive to changing conditions. A profound knowledge of the relevant atmospheric parameters at different locations is needed for the development of these adaptive systems and has to be obtained from measurements.

The papers published in this special section deal with all of these aspects. Some of the papers address more theoretical aspects, such as the propagation of Bessel beams and conic waves in a turbulent atmosphere and how this propagation influences the coherence of these beams. Another topic is a method for the efficient synthesis of imagery disturbed by turbulence with varying turbulence parameters (e.g. Fried's coherence diameter radius). In contrast to other approaches, this method uses dictionaries of turbulence characteristics to construct directly point spread functions in the image plane. The simulated imagery can serve as input during the development phase of algorithms for the adaptive correction of these disturbances.

Another paper focuses on work that is more experimental in nature. Here, the effect of aerosols on the quality of remotely acquired imagery is investigated. An image of a binary knife-edge target is recorded with a water droplet cloud chamber between the target and the camera. The modulation transfer function is derived experimentally and compared to theoretical calculations for different droplet size distributions and particle densities using common aerosol scattering models.

Practical implementation of adaptive optics components is described in two papers. One of these papers focuses on the development of elements for an adaptive optics system to be implemented on a solar telescope of the Siberian branch of the Russian Academy of Sciences. A special version of a wavefront sensor based on the Shack-Hartmann approach is described, which allows for a fast change of the lenslet arrays used. This facilitates a fast adaptation to prevailing turbulence conditions. The second paper discusses a potential solution to the problem of gaining a space situational awareness (SSA) picture using ground-based telescopes. Current approaches are costly and enable the surveillance of a small fraction of the sky only. In spite of problems with the optical quality of carbon fiber reinforced polymer due to the fabrication process, telescopes using these mirrors might enable cost-effective and field-deployable systems for the realization of SSA. It is shown that high-resolution imagery can be obtained if the telescope is equipped with a wavefront sensor (WFS) and the recorded images are processed simultaneously with the WFS data by an appropriate image restoration algorithm. 